\title{
Effects of robot viscous forces on arm movements in chronic stroke survivors: a randomized crossover study
}

Yazan Abdel Majeed ${ }^{1,3+}\left(\mathbb{0}\right.$, Saria Awadalla ${ }^{2+}$ and James L. Patton ${ }^{1,3^{*+}}$

\begin{abstract}
Background: Our previous work showed that speed is linked to the ability to recover in chronic stroke survivors. Participants moving faster on the first day of a 3-week study had greater improvements on the Wolf Motor Function Test.

Methods: We examined the effects of three candidate speed-modifying fields in a crossover design: negative viscosity, positive viscosity, and a "breakthrough" force that vanishes after speed exceeds an individualized threshold.

Results: Negative viscosity resulted in a significant speed increase when it was on. No lasting after effects on movement speed were observed from any of these treatments, however, training with negative viscosity led to significant improvements in movement accuracy and smoothness.
\end{abstract}

Conclusions: Our results suggest that negative viscosity could be used as a treatment to augment the training process while still allowing participants to make their own volitional motions in practice.

Trial registration: This study was approved by the Institutional Review Boards at Northwestern University (STU00206579) and the University of Illinois at Chicago (2018-1251).

Keywords: Stroke, Reaching, Speed, Viscosity, Crossover

\section{Background}

Stroke neurorehabilitation often uses the unique aspects of technology to improve motor recovery. While some researchers endeavored to simply assist movement to more closely resemble healthy patterns [1-3], others have attempted to exploit unique capabilities of robotics or graphic feedback to encourage neuroplasticity by augmenting error [4-8]. Even some traditional physical therapy exercises use mirrors to get the paretic side of the body to imitate the non-paretic side [9]. These are beneficial but far from a complete cure, and it remains to be

\footnotetext{
*Correspondence: pattonj@uic.edu

†Yazan Abdel Majeed, Saria Awadalla and James L. Patton contributed equally to this work

${ }^{1}$ Richard and Loan Hill Bioengineering Department, University of Illinois at Chicago, Morgan St, 60607 Chicago, USA

Full list of author information is available at the end of the article
}

seen what strategies emerge as optimal and what might still be left undiscovered.

An alternative strategy is to first uncover the attributes associated with better clinical movement outcomes, and then target training around these [10, 11]. Our previous work [12] employed a data-driven approach to model participant improvement using metrics derived from the movements themselves. We found that participant movement speed during the initial evaluation was most predictive of clinical changes. This speed was also the most strongly correlated with changes in the Wolf Motor Function Test (WMFT), making heightened speed a possible intervention for stroke. However, before such an intervention might be tested in clinical trials, we need to establish effective methods for speeding up participants.

There are multiple possible training conditions that may achieve this increase, and here we compare three candidate classes of conditions. One approach to affect original author(s) and the source, provide a link to the Creative Commons licence, and indicate if changes were made. The images or other third party material in this article are included in the article's Creative Commons licence, unless indicated otherwise in a credit line to the material. If material is not included in the article's Creative Commons licence and your intended use is not permitted by statutory regulation or exceeds the permitted use, you will need to obtain permission directly from the copyright holder. To view a copy of this licence, visit http://creativecommons.org/licenses/by/4.0/. The Creative Commons Public Domain Dedication waiver (http://creativeco mmons.org/publicdomain/zero/1.0/) applies to the data made available in this article, unless otherwise stated in a credit line to the data. 
movement speed is to directly increase it with a negative viscous field; previous work [13-16] showed that training with negative viscosity can improve participant movement and movement generalization abilities. Another possibility is to leverage the motor control mechanisms of error augmentation and after effects. Under this paradigm, participants would train with positive viscosity, under the expectation that their speed would increase as an aftereffect of that training when these resistive forces are removed [6, 17]. Finally, some research has shown that combining a resistive paradigm with a reward mechanism [18] may help participants learn better. In this case, participants will move in a positive viscosity field that attempts to slow them down, but moving above a certain speed is rewarded by a "breakthrough" where resistance vanishes. Participants may bias movements towards higher speeds to avoid the resistance.

Though it is somewhat understandable why these training conditions would change participant movement speed, the change being an increase is less obvious, especially when it comes to positive viscosity and breakthrough. Many research studies have demonstrated that training under certain conditions that alter normal movement - or perception of it - will induce an aftereffect in the opposite direction. This was shown to be true for primates [19] and humans [20]. Our reasoning for including positive viscosity is that the aftereffect of slowing movement speed would be an increase in speed when the slowing forces are removed. The breakthrough condition leverages the idea of limit-push. There is some evidence that introducing a "penalty" for participant movements that are undesired, and removing that penalty when participants conform to desired movements will bias subsequent motion towards these desired patterns, this was demonstrated using robotic forces [21] and purely visual distortions [22]. By penalizing slower movements in our breakthrough condition, and rewarding faster movements with removal of that penalty, we hope to bias participant motion towards these higher speeds.

In this preliminary clinical study, we simply compared the effects of these three paradigms on participant speed. Our modest goal was to determine if it was possible to influence participants' speed. If speed $i s$ something that can be changed, we will explore the more difficult question of its influence on functional recovery for a later trial. Chronic stroke survivors participated in a singlevisit crossover trial, where they trained for a short time under these three conditions. While we were mainly interested in the direct- and after-effects of these force paradigms on participant movement speed, we examined their effects on other movement metrics as well, such as error, efficiency, and smoothness.

\section{Methods}

\section{Participant population}

We recruited 14 chronic hemiparetic stroke survivors (one participant was not able to take part in the study after consenting due to a second stroke), 15-50 on the Upper Extremity Fugl-Meyer scale. Participants were excluded if they had multiple lesions or multiple stroke events, if they had bilateral paresis, or if they had Botox ${ }^{\circledR}$ treatments to the upper limbs within the last six months. Stroke survivors were recruited through the Clinical Neuroscience Research Registry of the Shirley Ryan AbilityLab and provided informed consent. This study was approved by the Institutional Review Boards at Northwestern University (STU00206579) and the University of Illinois at Chicago (2018-1251), and follows the guidelines of the Declaration of Helsinki. Figure 1 shows a CONSORT-style diagram (CONsolidated Standards Of Reporting Trials) for our crossover study, and Table 1 shows participant information at the time of participation in the study.

\section{Experiment design}

Participants completed a single-visit crossover study. Participants performed a targeted reaching task with their paretic arm attached to the Proficio ${ }^{\circledR}$ 3-DoF robot from Barrett Technologies (Fig. 2). The Proficio allows three dimensional movement in a large workspace, approximating the normal range of human motion.

Participants started from a neutral home position that was customized for each person. Each trial consisted of a center-out motion to a spherical target with $3 \mathrm{~cm}$ diameter, $15 \mathrm{~cm}$ away. One of eight possible targets was displayed at random for each center-out reach. Participants then returned to the home position and the next target was displayed. Participants had $10 \mathrm{~s}$ to complete each trial, after which the trial ends, and the participants return to the home position.

First, we evaluated baseline performance. Participants reached for five minutes to targets distributed in a quarter-sphere under no robotic forces. Participants then alternated between five minutes of reaching under each of the three experimental conditions, and five minutes of reaching with no forces to evaluate the aftereffects. The parts of the trial involving reaching under robotic forces did not include any "catch" trials where participants did not receive forces. This block design provided consistent rest periods, and consistent chunks of data for statistics. It also allows sufficient time for the effects of each force type to wash out before the next forces were presented. We presented the three conditions to each participant in a pseudorandom order. "Pseudo-random" refers to randomizing what each participant experienced while ensuring that 
Table 1 Participant information for this study $(N=14)$

\begin{tabular}{|c|c|c|c|c|c|c|}
\hline Participant ID & Age (years) & $\begin{array}{l}\text { Time since stroke } \\
\text { (months) }\end{array}$ & Sex & Dominant side & Affected side & UEFM score \\
\hline SP01 & 59 & 29 & M & Right & Right & 26 \\
\hline SP02 & 52 & 70 & M & Right & Left & 44 \\
\hline $\mathrm{SP} 03^{\mathrm{a}}$ & 64 & 29 & M & Right & Right & 39 \\
\hline SPO4 & 59 & 45 & M & Left & Right & 37 \\
\hline SP05 & 37 & 52 & M & Right & Right & 32 \\
\hline SP06 & 64 & 95 & M & Right & Left & 35 \\
\hline SP07 & 83 & 32 & M & Right & Right & 46 \\
\hline SP08 & 45 & 75 & $\mathrm{~F}$ & Right & Left & 23 \\
\hline SP09 & 67 & 11 & M & Right & Left & 37 \\
\hline SP10 & 50 & 157 & $\mathrm{~F}$ & Right & Left & 33 \\
\hline SP11 & 58 & 50 & $\mathrm{~F}$ & Right & Right & 40 \\
\hline SP12 & 57 & 29 & $\mathrm{~F}$ & Right & Left & 24 \\
\hline SP13 & 67 & 49 & M & Right & Right & 23 \\
\hline SP14 & 46 & 43 & $\mathrm{~F}$ & Right & Left & 34 \\
\hline
\end{tabular}

a SP03 consented but did not perform the study due to a second stroke

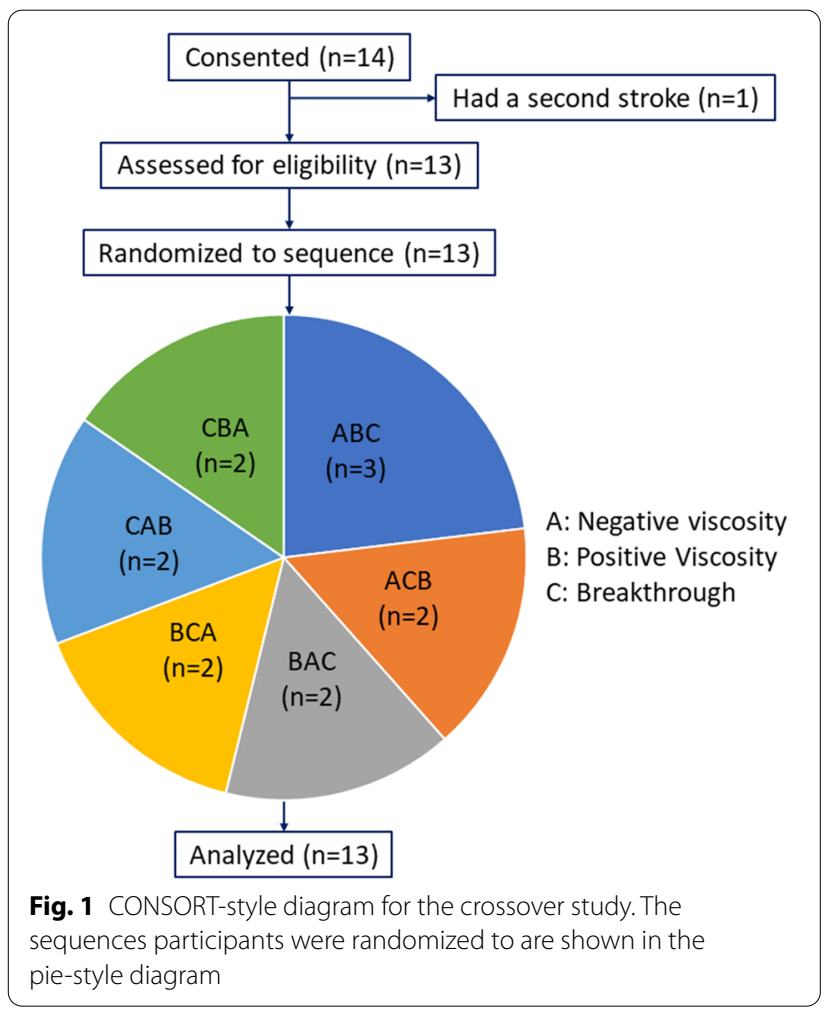

we had at least 2 participant experiencing each of the 6 possible force orders, to control for any possible ordering effect. A simplified 1-D representation of the three force types for our experimental conditions is shown in Fig. $3 \mathrm{a}-\mathrm{c}$ and the timeline of the experiment is shown in Fig. 3d.

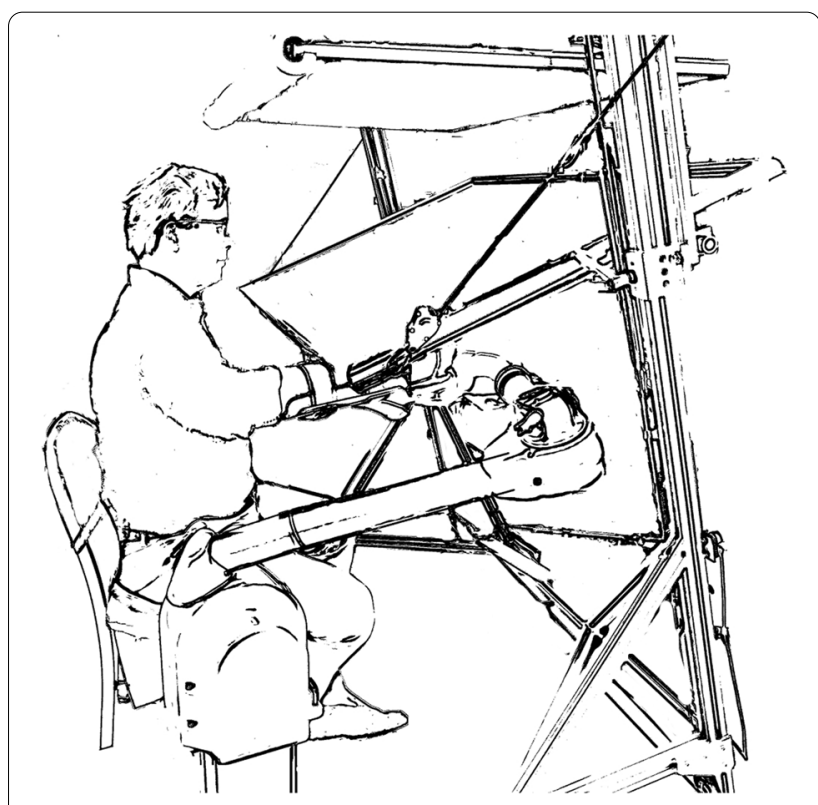

Fig. 2 Experiment setup. Participants reached unimanually, alternating between a central home position and randomly to one of eight different target locations. The study was conducted using the Looking Glass virtual reality system and the Barrett Proficio robot arm

\section{Data analysis}

We resampled participant position data (originally at $1 \mathrm{kHz}$ ) to $100 \mathrm{~Hz}$, then filtered it using a 7th order Butterworth filter with a $9 \mathrm{~Hz}$ cutoff frequency. We extracted the following metrics from each movement, a lot of which are also explained in our previous paper [12]: 

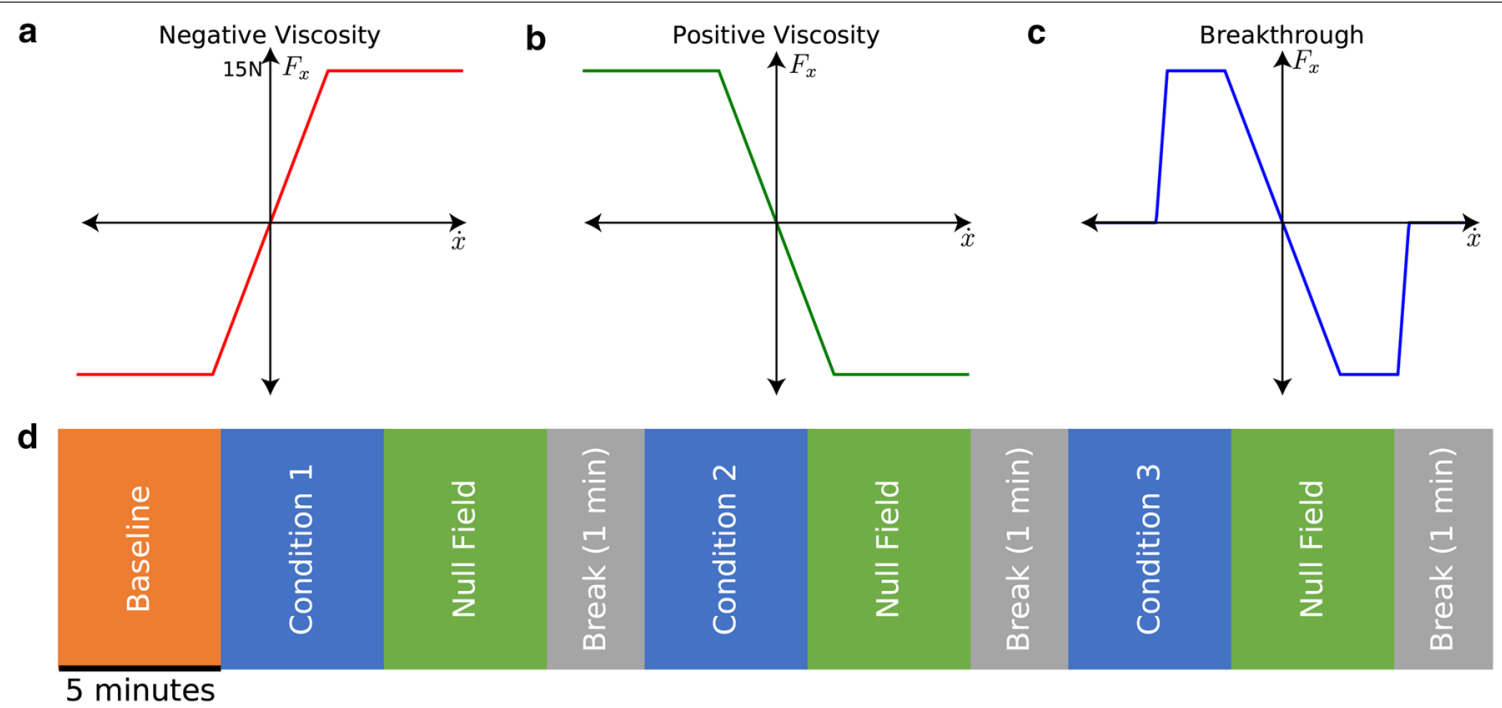

Fig. 3 Experiment conditions and timeline a Timeline of the experiment, each "block" of trials lasted for 5 min, and there was a short break (30-60 s) in after each block. b Negative viscosity. Forces were proportional to velocity in all three directions, we drove the forces to zero at higher speeds for safety. $\mathbf{c}$ Positive viscosity. Similar to negative viscosity but the forces acted opposite to the direction of motion, slowing the participants. $\mathbf{d}$ Breakthrough. Forces were proportional to velocity until the participants reached $75 \%$ of their baseline speed, forces were then removed as a reward for reaching faster speeds

- Movement accuracy (max perpendicular distance): Participants were instructed to reach to the targets in a straight line, our chosen error measure was the maximum distance from the straight line between the initial position when the target was shown to the position of the target.

- Max and mean speed: We extracted the maximum speed during each trial, and calculated the mean speed as total path length divided by trial time.

- Initial direction error: We calculated the initial direction error as the angle (in radians) between the straight line to the target and the line connecting the initial position to the position where the participant reached $10 \%$ of their maximum speed. This was calculated independently for each trial.

- Pre-movement speed: We defined pre-movement speed as the average speed before participants 'launched' their movement, defined as reaching 10\% of their maximum speed.

- Initial movement ratio: Ratio of the distance covered during the first part of the movement (defined as the first speed peak, before making corrections) and the total movement distance.

- Movement efficiency (path length ratio): Ratio of the total movement distance and the straight line distance between the initial position and the target.

- Movement smoothness (number of submovements): We approximated the number of submovements as the number of speed peaks during each trial.
- Speed ratio: Ratio of the participant's launch speed (speed of the first peak) and the maximum speed during the trial.

- Percentage of movement in the target direction: We calculated this metric by projecting the position vector between each pair of consecutive time samples onto the straight line from start to target, summing up the projections, and finally dividing by the hand path length.

- Arrest period ratio: The percentage of time during a trial where the participant was moving slower than $10 \%$ of max trial speed.

We focused our analysis for each condition (robot viscous force type) on groups of five trials: prior to exposure to the robotic forces (pre-exposure/baseline), when the forces were initially experienced (early exposure), at the end of the five minute block experiencing the forces (late exposure), the reaction to the forces being turned off (aftereffects), and the end of the null field block before the next condition is experienced (retention). We modeled study outcomes using linear mixed effects regression of the fixed conditions (vs baseline), time, and random subject effects.

$$
y=x * \beta+Z * u+\epsilon
$$

where $y$ represents the metric we are exploring, for example maximum speed, $X$ represents the fixed effects (force condition, trial number), and $Z$ represents the random 
effects (participant ID). We also made sure to check whether there was an interaction between the two fixed effects had an influence on the outcome, or whether the order of presenting the force conditions had an effect.

We compared differences between condition pairs using post-hoc contrasts with Tukey's adjustment for multiple comparisons in all cases. This was done regardless of whether the initial p-values were significant. We did not assume data normality in our mixed effects model or our statistical tests, however, since we had $>250$ trials in each phase of the study, we are leveraging the central limit theory and showing means and standard deviations in Figs. 4 and 5.

\section{Results}

\section{Recruitement and participant issues}

We enrolled one additional participant in case parts of the movement data from the first participant was unusable. Due to a participant initially having difficulty stopping their motion due to weakness in counteracting the negative viscosity forces. We were ultimately able to utilize the data we collected from all participants, and had a grand total of 13 participants.

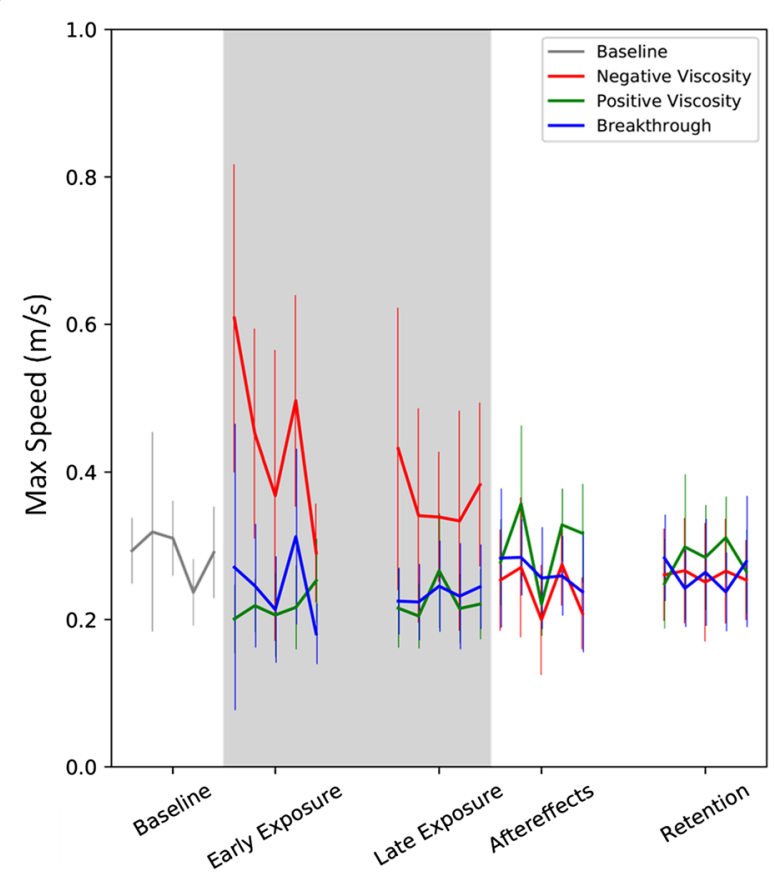

Fig. 4 Effect of experiment conditions on altering participant speed. Thick lines represent means. Error bars represent 95\% confidence intervals across all participants. Each point on the thick lines is a trial. Robot forces were turned on in the shaded region

\section{Movement speed}

Negative viscosity produced significant speed increases during early exposure that persisted through late exposure $(p<0.0004$, Fig. 4$)$. There was a significant difference between the effect of negative and positive viscosity forces $(p<0.001)$, and between negative viscosity and breakthrough forces $(p<0.03)$ during both early and late exposure. The order with which the force conditions were presented to the participants did not have a significant effect on speed $(p>0.16)$. The aftereffect of negative viscosity was a significant decrease in speed $(p<0.02)$ which did not persist at the end of washout (retention $p>0.09$ ).

During early exposure, neither breakthrough nor positive viscosity had any significant effects on movement speed $(p>0.11)$. Both had a significant slowing effect on maximum speed $(p<0.03)$ but not on mean speed $(p>0.055)$ during late exposure. Neither had any significant effect on speed during washout $(p>0.07)$. There was no significant difference in the effects of positive viscosity and breakthrough forces during any stage of the experiment $(p>0.48)$. The effects of negative and positive viscosity forces were significantly different $(p<0.02)$ for all but the retention stage $(p>0.61)$.

\section{Other movement metrics}

Movement error increased significantly during early exposure to negative viscosity $(p=0.004$, Fig. 5A). The effect was no longer significant by late exposure $(p=0.064)$. However, both the aftereffect and retention had a significant reduction in movement error $(p<0.023)$ compared to the baseline phase. Training with positive viscosity forces yielded a significant increase in movement error during exposure $(p<0.01)$ but no significant aftereffects. Breakthrough forces had no significant effect on error during exposure, but showed a significant error reduction as an aftereffect $(p<0.02)$. Interestingly, the retention movement error was affected by the order the force types were presented to participants $(p=0.04)$, other stages were not affected by the order $(p>0.06)$.

Movement distance showed behavior similar to movement error (Fig. 5b), where negative viscosity resulted in worse performance during exposure $(p<0.0001)$ but significantly improved performance as an after effect $(p \leq 0.001)$. Positive viscosity had no significant effects during any stage, while breakthrough showed a significant improvement only during the aftereffect $(p \leq 0.001$ ) .

Movement smoothness, as quantified by the number of speed peaks, was significantly worse during exposure to negative viscosity $(p \leq 0.0099)$ and significantly 

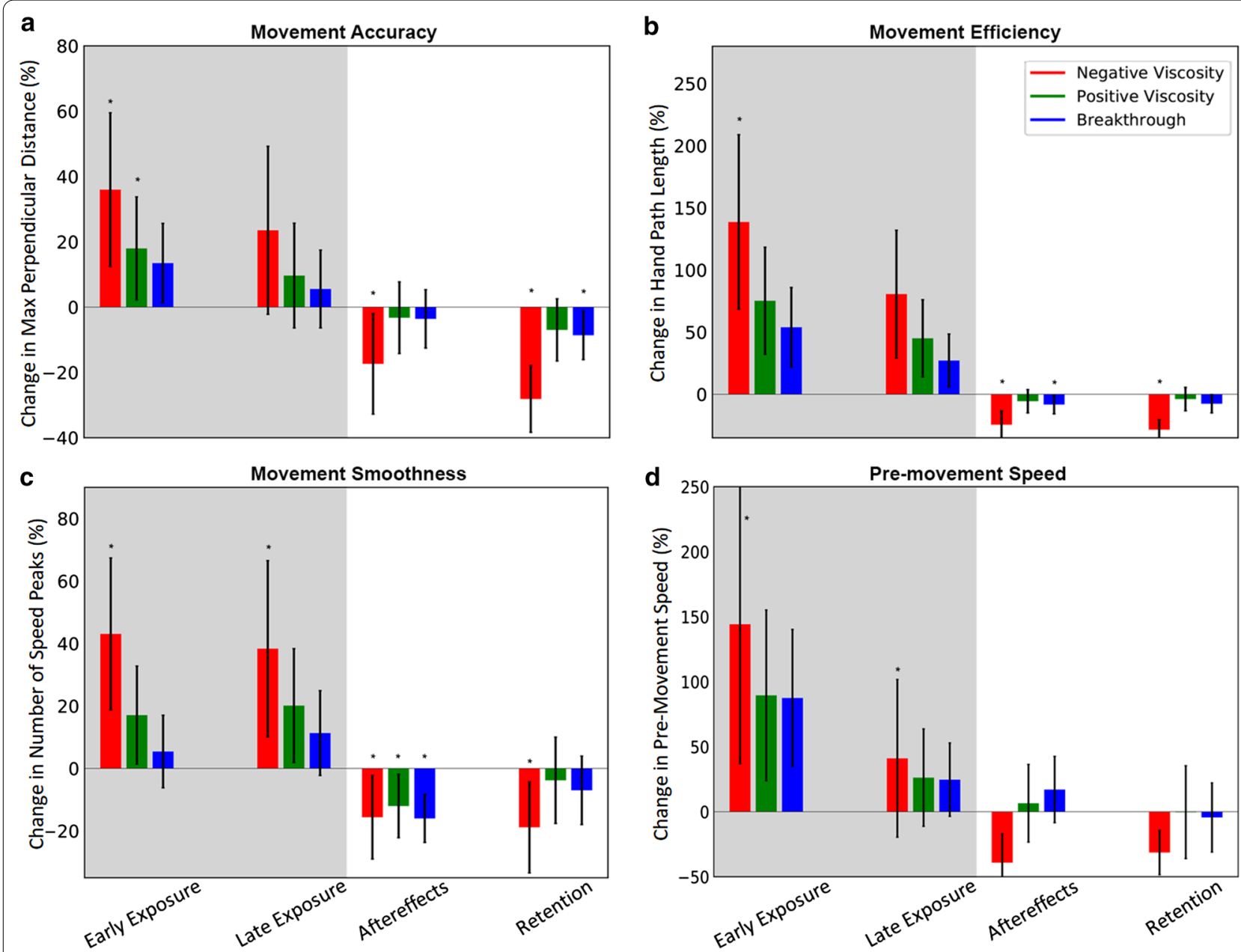

Fig. 5 Effect of experiment conditions on various movement features. a Movement accuracy. b Movement efficiency. c Movement smoothness. d Pre-movement speed. Participants experienced robot forces in the shaded regions. The zero line represents the average of each metric during the baseline block, independently for each participant

better during washout ( $p \leq 0.033$, Fig. 5c) compared to baseline. Interestingly, all force types showed a significant improvement in movement smoothness in early aftereffects $(p \leq 0.031)$, though only after training with negative viscosity did participants retain that improvement.

Finally, there was a significant increase in premovement speed during exposure to negative viscosity (Fig. 5d). There was no significant effect for any of the three force types on this pre-movement speed by late washout, though training with negative viscosity showed an average reduction in pre-movement speed.

There were other smaller, mostly non-significant effects of the other force conditions on these movement metrics. Overall, participants did not improve their reaches after training with positive viscosity or breakthrough forces.

\section{Discussion}

We looked for evidence of speeding participants up in both the direct effects when we turn on robotic forces and after effects after minutes of exposure then turning the forces off. As we expected, negative viscosity produced the largest direct effect (an increase in speed), since it was directly pushing participants to move faster. Speeds decreased by the end of exposure as they adapted, although they remained faster than baseline - a prolonged direct effect. Interestingly, we observed no speedrelated after effects from any of our force treatments and any mismatch from baseline behaviour quickly dissipated.

While participants were reaching under the positive viscosity condition, they exhibited slower movements as we expected, though this slowdown was not statistically significant in most cases. There was a minor increase in movement speed as an aftereffect, which was also not significant. Ultimately, participants behaved as we 
expected during and after experiencing positive viscosity, but the effects were very small compared to their baseline movements.

We expected that breakthrough forces would bias towards faster movements during late exposure and the early aftereffect due to this condition rewarding faster movements. We instead saw a reduction in movement speed. This can be due to incomplete learning of the forces, where participants did not have enough experience with the breakthrough condition to sufficiently bias their movements. Another explanation can be that breakthrough forces do not have the desired effect on participant movement speed. Either way, in a direct comparison with negative and positive viscosity under similar force magnitudes and length of exposure, breakthrough forces did not succeed in increasing participant speed.

Negative viscosity reduces participants' ability to control their arm movements during the initial ballistic phase. This led to a significant reduction in movement accuracy, effectiveness, and smoothness, as participants tried to counteract the destabilising force. These effects were reversed, as expected, once the forces were turned off, and participants significantly improved their reaches.

Although our long-term goals are to provide better rehabilitation (transferring to recovery and functional improvement), the goals of this paper were more modest, to determine if it is possible to influence participant speed. The goals of testing this in a protracted intervention will be tested in a later study. It remains to be seen whether increased speed from our work also has an effect.

We expected incomplete learning and/or incomplete washout due to our short exposure and null field blocks. Hence, we presented the forces to our participants in a randomized order and tested for any ordering effect. Out of 30 linear mixed effects models, the order the forces were experienced was significant in only two cases: late exposure movement distance, and retention movement error. This could be due to a few outliers in our small sample of participants, since in both cases the p-values were just below the significance level $(p \approx 0.04)$.

Our goal with a crossover design was to elucidate the effects three robotic forces with heterogeneous stroke subjects, but there are intrinsic limitations with a crossover study. First, there may be an order (carryover) effect that can obscure the effects of any one treatment. In randomizing the order into all possible presentations (6 groups), we did our best at dealing with this. With $3 !=6$ possible order sequences for the three force types, we wanted to examine data from at least two participants per order sequence (1). Another limitation of crossover designs is the reduction in statistical power. This pilot study was not prospectively powered for significant outcomes due to lack of preliminary data. In spite of this, our work provided significant positive outcomes for some of the conditions (negative viscosity), which should guide future studies that employ this type of speed interventions. A third limitation is increased subject dropout, which was luckily not a problem in our case.

It is paradoxical that a speed-dependent force field that enhances velocities might also exacerbate spasticity or rigidity of the arm. Spasticity is defined as a hyperexcitable velocity-dependent stretch reflex [23, 24] and is typically measured with the modified Ashworth scale (MAS). Other researchers have shown that progressive reflex increases with repetitive stimuli (i.e., "windup") in patients [25]. These repetitive stimuli may have different effects on the arm's spasticity response depending on where the patient falls on the MAS. We happened to only get participants who did not have significant contractures or an MAS score of more than 3, as assessed by an occupational therapist. Modified Ashworth, the measure of spasticity, was not controlled for in this study, so it remains to be seen whether spastic participants are more or less likely to respond to our speed-enhancement in training.

Since we were conditioning the arm, repeated exposure to a stimulus that causes spastic responses may have a therapeutic effect. Training with velocity-enhancing fields showed that negative viscosity can improve participant movement and movement generalization abilities [13-16]. Our work here also did not definitively address the responsiveness to prolonged speed training, where spastic post-stroke patients may respond differently to such therapy. Interestingly, Park, et al. (NNR 2016) [26] also showed that training that emphasizes fast movements (therefore high speeds) leads to large improvements, which bolsters the hypothesis that training with negative viscosity may improve patient performance. Their (unassisted) training method led to significant fatigue [27]. We believe that fatigue is not an issue with our paradigm, since the patients do not have to support the weight of their arm against gravity. The robot performs gravity compensation thereby significantly reducing fatigue while performing the reaching task.

The rehabilitation literature supports that a more demanding training protocol leads to faster learning and improvements [28-31]. This study was impossible to control for this, as speed is part of any energetic calculation [32], but difference in metabolic cost across conditions may also influence outcome. It may be, that although speed was a target and proved to be influenced, the more critical aspect might be Work mechanical work or calories burned. If so it may also be that enhancing velocity is a good way to accomplish this. 
Negative damping has been compared to methods that enhance error for rehabilitation [5, 6], though this was not our elicit goal. The reasoning is that any mistake is often made larger by negative viscosity. Error based learning leverages one of the well-known neurocomputational mechanisms of plasticity - supervised learning. This excludes the two other methods, reward-based reinforcement and repetitive strengthening of Hebbian. Erroraugmentation paradigm, positive reinforcement is best suppressed so that error mechanisms can drive change in neural connections [33, 34]. Nevertheless, enhancing speed during training might be a way to simply give more experience (both errors or successes).

This study showed that negative viscosity had a strong direct effect at increasing participant speed. Participants also demonstrated improvements in movement accuracy, efficiency, and smoothness after the training. These findings are encouraging, and bolster the potential for using viscosity for clinical treatment. We are currently piloting a clinical exploration that uses negative viscosity to train chronic stroke survivors over multiple sessions. Our hypothesis is that we will see improvements in performance on standard clinical assessments, as a prelude to conducting a full clinical trial.

\section{Conclusions}

In a direct comparison between the three force conditions that may increase movement speed, participants significantly increased their speed only as a direct effect of negative viscosity. Positive viscosity and breakthrough forces had no effect on participant speed. After the forces were removed, only negative viscosity showed significant improvements in other movement metrics measuring accuracy, efficiency, and smoothness. We conclude that training to increase movement speed should be conducted using negative viscosity. Even though we did not achieve the desired lasting effect on movement speed, the improvements in other movement parameters shows promise for negative viscosity as a potential treatment.

\section{Abbreviations}

UEFM:: Upper extremity Fugl-Meyer; WMFT:: Wolf motor function test; MAS:: Modified Ashworth scale.

\section{Acknowledgements}

We would like to thank Jennifer Kahn, PT/DPT/NCS at the Shirley Ryan AbilityLab for her help in accessing and using the Clinical Neuroscience Research Registry (STU00013948). We would like to also thank Courtney Celian, OTR/L for her help in recruiting participants for this study.

\section{Authors' contributions}

YAM collected, analyzed, and interpreted the data, and wrote the initial manuscript. SA worked on and provided direction for the statistical analysis. JLP directed the research and study design. All authors edited and reviewed the manuscript.

\section{Funding}

The research presented in this article was supported by the American Heart Association (Predoctoral Fellowship 18PRE34080333) and the Shirley Ryan AbilityLab. Funding entities were not involved in data collection, analysis, or interpretation beyond providing compensation for the participants and study team.

\section{Availability of data and materials}

The datasets used and/or analysed during the current study are available from the corresponding author on reasonable request.

\section{Ethics approval and consent to participate}

This study was approved by the Institutional Review Boards at the University of Illinois at Chicago (2018-1251) and Northwestern University (STU00206579). All members of the research team were approved for conducting research with human participants.

\section{Consent for publication}

Not applicable.

\section{Competing interests}

The authors declare that they have no competing interests.

\section{Author details}

${ }^{1}$ Richard and Loan Hill Bioengineering Department, University of Illinois at Chicago, Morgan St, 60607 Chicago, USA. ${ }^{2}$ School of Public Health, University of Illinois at Chicago, Taylor St, 60612 Chicago, USA. ${ }^{3}$ Shirley Ryan AbilityLab, Erie St, 60611 Chicago, USA.

Received: 18 December 2019 Accepted: 29 October 2020

Published online: 24 November 2020

\section{References}

1. Schabowsky CN, Godfrey SB, Holley RJ, Lum PS. Development and pilot testing of HEXORR: hand EXOskeleton rehabilitation robot. J Neuroeng Rehabil. 2010;7(1):36.

2. Fisher Bittmann MF. Customized robotic training approaches using the statistics of reaching errors. University of Illinois at Chicago; 2016.

3. Konidaris G, Kuindersma S, Grupen R, Barto A. Robot learning from demonstration by constructing skill trees. Int J Robot Res. 2012;31(3):360-75.

4. Wright ZA, Lazzaro E, Thielbar KO, Patton JL, Huang FC. Robot training with vector fields based on stroke survivors' individual movement statistics. IEEE Trans Neural Syst Rehabil Eng. 2017;26(2):307-23.

5. Fisher ME, Huang FC, Klamroth-Marganska V, Riener R, Patton JL, Haptic error fields for robotic training. In: IEEE World Haptics Conference (WHC). IEEE. 2015;2015:434-9.

6. Abdollahi F, Case Lazarro ED, Listenberger M, Kenyon RV, Kovic M, Bogey RA, et al. Error augmentation enhancing arm recovery in individuals with chronic stroke: a randomized crossover design. Neurorehabil Neural Repair. 2014;28(2):120-8.

7. Patton JL, Huang FC. Sensory-motor interactions and error augmentation. Neurorehabilitation technology. Berlin: Springer; 2016. p. 79-95.

8. Marchal-Crespo L, Michels L, Jaeger L, López-Olóriz J, Riener R. Effect of error augmentation on brain activation and motor learning of a complex locomotor task. Front Neurosci. 2017;11:526.

9. Altschuler EL, Wisdom SB, Stone L, Foster C, Galasko D, Llewellyn DME, et al. Rehabilitation of hemiparesis after stroke with a mirror. Lancet. 1999;353(9169):2035-6.

10. Mostafavi SM, Glasgow Jl, Dukelow SP, Scott SH, Mousavi P. Prediction of stroke-related diagnostic and prognostic measures using robot-based evaluation. In: 2013 IEEE 13th International Conference on Rehabilitation Robotics (ICORR). IEEE; 2013. p. 1-6.

11. Yu L, Xiong D, Guo L, Wang J. A remote quantitative Fugl-Meyer assessment framework for stroke patients based on wearable sensor networks. Comput Methods Programs Biomed. 2016;128:100-10.

12. Majeed YA, Awadalla SS, Patton JL. Regression techniques employing feature selection to predict clinical outcomes in stroke. PloS One. 2018;13(10):e0205639. 
13. Dancause N, Ptitob A, Levin MF. Error correction strategies for motor behavior after unilateral brain damage: short-term motor learning processes. Neuropsychologia. 2002;40(8):1313-23.

14. Huang FC, Patton JL. Augmented dynamics and motor exploration as training for stroke. IEEE Trans Biomed Eng. 2012;60(3):838-44.

15. Israely S, Carmeli E. Error augmentation as a possible technique for improving upper extremity motor performance after a stroke-a systematic review. Topics Stroke Rehabil. 2016;23(2):116-25.

16. Patton JL, Kovic M, Mussa-Ivaldi FA. Custom-designed haptic training for restoring reaching ability to individuals with poststroke hemiparesis. J Rehabil Res Dev. 2006;43(5):643-56.

17. Patton JL, Stoykov ME, Kovic M, Mussa-Ivaldi FA. Evaluation of robotic training forces that either enhance or reduce error in chronic hemiparetic stroke survivors. Exp Brain Res. 2006;168(3):368-83.

18. Shah AK, Sharp I, Hajissa E, Patton JL. Reshaping movement distributions with Limit-Push robotic training. IEEE Trans Neural Syst Rehabil Eng. 2018;26(11):2134-44.

19. Yin PB, Kitazawa S. Long-lasting aftereffects of prism adaptation in the monkey. Exp Brain Res. 2001;141(2):250-3.

20. Mistry M, Mohajerian P, Schaal S. An exoskeleton robot for human arm movement study. In: 2005 IEEE/RSJ international conference on intelligent robots and systems. IEEE; 2005. p. 4071-4076.

21. Sharp I, Patton JL. Limit-push training reduces motor variability. In: 2011 IEEE International conference on rehabilitation robotics. IEEE; 2011. p. 1-6.

22. Hajissa E, Shah A, Patton JL. Visual limit-push training alters movement variability. IEEE Trans Biomed Eng. 2017;65(10):2162-7.

23. Lance JW. What is spasticity?[comment]. Lancet. 1990;335(8689):606.

24. Lance JW, Burke D. Mechanisms of spasticity. Arch Phys Med Rehabil. 1974;55(8):332-7.

25. Hornby T, Rymer W, Benz E, Schmit B. Windup of flexion reflexes in chronic human spinal cord injury: a marker for neuronal plateau potentials? J Neurophysiol. 2003;89(1):416-26.

26. Park H, Kim S, Winstein CJ, Gordon J, Schweighofer N. Short-duration and intensive training improves long-term reaching performance in individuals with chronic stroke. Neurorehabil Neural Repair. 2016;30(6):551-61.

27. Park H, Schweighofer N. Nonlinear mixed-effects model reveals a distinction between learning and performance in intensive reach training post-stroke. J Neuroeng Rehabil. 2017;14(1):21.

28. Kwakkel G. Impact of intensity of practice after stroke: issues for consideration. Disability Rehabil. 2006;28(13-14):823-30.

29. Emken JL, Benitez R, Sideris A, Bobrow JE, Reinkensmeyer DJ. Motor adaptation as a greedy optimization of error and effort. J Neurophysiol. 2007;97(6):3997-4006.

30. Lohse KR, Lang CE, Boyd LA. Is more better? Using metadata to explore dose-response relationships in stroke rehabilitation. Stroke. 2014;45(7):2053-8.

31. Reinkensmeyer DJ, Burdet E, Casadio M, Krakauer JW, Kwakkel G, Lang CE, et al. Computational neurorehabilitation: modeling plasticity and learning to predict recovery. J NeuroEng Rehabil. 2016;13(1):42.

32. Wright ZA, Patton JL, Huang FC, Energetics during robot-assisted training predicts recovery in stroke. In: 40th Annual international conference of the IEEE engineering in medicine and biology society (EMBC). IEEE. 2018;2018:2507-10

33. Marchal-Crespo L, Reinkensmeyer DJ. Review of control strategies for robotic movement training after neurologic injury. J Neuroeng Rehabil. 2009;6(1):1-15.

34. Ballester BR, Nirme J, Duarte E, Cuxart A, Rodriguez S, Verschure P, et al. The visual amplification of goal-oriented movements counteracts acquired non-use in hemiparetic stroke patients. J Neuroeng Rehabi. 2015;12(1):50.

\section{Publisher's Note}

Springer Nature remains neutral with regard to jurisdictional claims in published maps and institutional affiliations.
Ready to submit your research? Choose BMC and benefit from:

- fast, convenient online submission

- thorough peer review by experienced researchers in your field

- rapid publication on acceptance

- support for research data, including large and complex data types

- gold Open Access which fosters wider collaboration and increased citations

- maximum visibility for your research: over 100M website views per year

At BMC, research is always in progress.

Learn more biomedcentral.com/submissions 